
\title{
25 Research Soure \\ Effects of strontium ions with potential antibacterial activity on in vivo bone regeneration
}

\section{Nafiseh Baheiraei ( $\square$ n.baheiraei@modares.ac.ir)}

Tarbiat Modares University Faculty of Medical Sciences https://orcid.org/0000-0002-8760-5971

\section{Hossein Eyni}

Tarbiat Modares University Faculty of Medical Sciences

\section{Bita bakhshi}

Tarbiat Modares University Faculty of Medical Sciences

\section{Raziyeh Najafloo \\ Tarbiat Modares University}

\section{Research article}

Keywords: Bioactive glass, Bone tissue engineering,Strontium, Osteogenesis

Posted Date: March 20th, 2020

DOl: https://doi.org/10.21203/rs.3.rs-18054/v1

License: (c) (1) This work is licensed under a Creative Commons Attribution 4.0 International License. Read Full License

Version of Record: A version of this preprint was published at Scientific Reports on April 22nd, 2021. See the published version at https://doi.org/10.1038/s41598-021-88058-1. 


\section{Abstract}

Background Bioactive glasses (BGs) have attracted added attention in the structure of the scaffolds for bone repair applications. Different metal ions could be doped in BGs to induce specific biological responses. Among these ions, strontium (Sr) is considered as an effective and safe doping element with promising effects on bone formation and regeneration. Methods In this experiment, we evaluated the antibacterial activities of the gelatin-BG (Gel-BG) and Gel-BG/Sr scaffolds in vitro. The osteogenic properties of the prepared scaffolds were also assessed in rabbit calvarial bone defects for 12 weeks. Alizarin Red, Hematoxylin \& Eosin (H\&E) and Masson's Trichrome staining were performed to assess bone regeneration and the obtained results were compared with those without Sr. Also, histomorphometric data were obtained to evaluate the new bone, residual graft, and connective tissue. Results Both scaffolds showed in vivo bone formation during 12 weeks with the newly formed bone area in Gel-BG/Sr scaffold was higher than that in Gel-BG scaffolds after the whole period. Based on the histological results, Gel-BG/Sr exhibited acceleration of early-stage bone formation in vivo. The results of antibacterial investigation showed that although both Gel-BG/Sr and Gel-BG effectively inhibited the growth of Escherichia coli (E. coli) but, only Gel-BG/Sr structure could lead to a 3 log reduction in Staphylococcus aureus (S. aureus). Conclusions: Our results confirmed that Sr doped BG is a favorable candidate for bone tissue engineering with superior antibacterial activity and bone regeneration capacity compared with similar counterparts having no Srion.

\section{Background}

Almost 1.3 million people experience bone graft surgeries due to the skeletal defects made by either accidents or diseases annually in the United States[1]. Obesity, genetic abnormalities, increased rate of accidents as well as the aging population are all considered as reasons which increase the number of bone lesions around the world[2]. Osteoporosis generated by decreased bone mineral density influences more than 200 million people worldwide, with half of this population undergoing a minimum one fracture during their lifetime[3]. Bone grafting either autografts or allografts are associated with limitations such as additional surgery, potential risk of transmitting diseases, immunological response, and long period issues[4]. Therefore, there is still a demand for developing safer and more effective alternatives. Bone tissue engineering is a promising strategy that aims to fabricate interconnected porous graft substitutes for bone defects reconstruction. Among material used for synthetic bone scaffolds, bioactive glasses (BGs) have attracted more attention in the structure of bone repair scaffolds in many investigations due to the properties such as osteogenesis, high level of bioactivity as well as the ability to bond with soft and hard tissues $[5,6]$ The synergistic effects of $\mathrm{Si}, \mathrm{Ca}$ and $\mathrm{P}$ ions released from $\mathrm{BG}$, could promote differentiation of osteoblasts via activation of osteogenesis-related signaling pathways[7]. Magnetic BGs are also reported having potential for hyperthermia treatment of malignant tumors, including bone cancer[8]. Interestingly, BGs have been clinically used to treat damages made by periodontal disease[9] as well as for ocular surgery applications[10]. 
Different metal ions could be doped in BGs to induce specific biological responses. Among these ions, strontium (Sr) is an alkaline earth metal which is presently utilized for the treatment of osteoporosis[11]. Strontium Renelate has also been stated to decrease the rate of fractures in elderly patients having osteoporosis[12] and has been clinically used to treat osteoporosis in postmenopausal patients[13]. Biomaterials containing Sr have been proved to enhance bone formation or/and remodeling[14]. Also, Sr is considered as an effective and safe doping element which its effect on bone formation and remodeling becomes more noticeable and different over time depending on the applied concentration[14]. This ion is reported to accelerate osteogenesis[15] and mineralization, as well[16]. The effects of Sr on bone healing and regeneration have been extensively studied in vitro and in vivo[17-19]. For example, Sr containing BG microspheres (Sr-BGM) have been shown to significantly improve early angiogenesis via modulating macrophages towards the $\mathrm{M} 2$ phenotype expressing a great value of platelet-derived growth factor-BB (PDGF-BB). The authors assumed that this early vascularization could efficiently promote new tissue regeneration, including bone formation[20]. Also, osteogenic capability of thermosensitive $\mathrm{p}(\mathrm{N}$ Isopropylacrylamide-co-butyl Methylacrylate) hydrogel (PIB nanogel) was increased significantly following the addition of mesoporous bioactive glass containing $\mathrm{Sr}$ (Sr-MBG). Scaffolds were inserted in rat femur defect two months after making the osteoporosis model. PIB nanogel was considered an excellent carrier for primary osteoblasts, which, together with Sr-MBG, improved the regeneration of the produced femur defects synergistically[3]. Addition of Sr and, or Li have also been shown to alter physicochemical properties of BG porous scaffolds, promoting osseointegration and bone remodeling in a rabbit femoral defect model[6]. The simultaneous effect of applying $\mathrm{Sr}$ and Co ions on the acceleration of bone healing and vascularization was further confirmed by implanting Sr-Co-BG seeded with human umbilical cord perivascular cells (HUCPVCs) in the knee defect of the rabbits for 12 weeks. The results revealed significant improved angiogenic and regeneration potential of BGs after being doped with $\mathrm{Sr}$ and $\mathrm{Co}[21]$. Previously, our group demonstrated that BG/Sr containing scaffolds promote proliferation and osteogenic differentiation of Mesenchymal Stem Cells (MSCs) as well as angiogenesis[17]. Here, we further evaluated the osteogenic properties of the gelatin-BG/Sr scaffolds in rabbit calvarial bone defects as well as their antibacterial features. The obtained results were compared with those without Sr.

\section{Materials And Methods}

Materials

All materials were purchased from Sigma- Aldrich (Germany) unless otherwise is specified.

Scaffold fabrication

Bioglass (BG) based on the $\mathrm{CaO}-\mathrm{SiO}_{2}-\mathrm{Na}_{2} \mathrm{O}-\mathrm{P}_{2} \mathrm{O}_{5}$ system and $\mathrm{BG}$ having strontium ( $\mathrm{BG} / \mathrm{Sr}$ ) in a $\mathrm{SiO}_{2}{ }^{-}$ CaO-SrO- $\mathrm{P}_{2} \mathrm{O}_{5}$ system was fabricated according to our previous protocol by sol- gel method with $\mathrm{Sr}$ was substituted for $\mathrm{Ca}$ at the percentage of $5 \mathrm{wt} \%[17]$. Scaffolds were prepared via freeze drying method, as previously explained[17]. Briefly, $15 \% \mathrm{w} / \mathrm{v}$ of the synthesized BG powder was added to the $5 \%(\mathrm{w} / \mathrm{v})$ aqueous solution of gelatin (Gel) and the obtained solution was then cast in a Teflon mold and frozen at 
$-20^{\circ} \mathrm{C}$ and $-80^{\circ} \mathrm{C}$ for 5 and $12 \mathrm{~h}$, respectively. The frozen samples were then lyophilized in a freeze drier (Alpha 1-4 LDplus, Martin Christ, Germany) for $48 \mathrm{~h}$ to fabricate porous scaffolds. The same instructions were used with BG/Sr powder to make the Gel- BG/Sr scaffolds ( $15 \% \mathrm{w} / \mathrm{v})$. Samples were cross linked using $0.5 \%$ glutaraldehyde for $24 \mathrm{~h}$, followed by excessive washing in deionized water for three days and further lyophilization.

Investigational animals and surgical procedures

Animal investigations were approved by the Ethics Committee of Tarbiat Modares University, Iran (IR.MODARES.REC.1398.070). Twelve New Zealand white rabbits of 2.6-3 kg in weight were used and randomly divided into three investigational groups ( $n=4 /$ group). A standard environment such as humidity, temperature, 12/12 hours light/dark, and standard food and water were applied before and after surgery. General anesthesia was accomplished with Xylazine (10 mg/Kg, $\%$, Alfasan, Woerden-Holland) and Ketamine ( $90 \mathrm{mg} / \mathrm{Kg}, 10 \%$, Alfasan, Woerden-Holland). Rabbits were prepared and draped using povidone iodide to sterilize the area. The calvaria were exposed by a skin incision ( $3 \mathrm{~cm}$ incision along the midline of the scalp) and then, muscle and periosteum were resected. Three standardized defects ( $8 \mathrm{~mm}$ in diameter) were formed in the parietal bones with low rotation speed of surgical trephine using sterile saline buffer to cool while clearing any residual debris. Two defects were filled with Gel-BG/Sr and Gel-BG scaffold, and the third one was left unfilled as a control group. Attention was taken to avoid displacement of the scaffolds into the other defects. At the end of the surgery, periosteum, muscle, and skin were repositioned and closed with absorbable VICRYL ® (Johnson \& Johnson Co., USA) sutures and Tetracycline (Iran Darou, Iran) was sprayed on calvaria. Amoxicillin $(0.1 \mathrm{ml} / \mathrm{kg}, 15 \%$, Tolide Darou, Iran) was also administrated intramuscularly to avoid infections. Animals were safe and kept warm in separate cages until recovery. They were then guided to the holding room and ordered Ketorolac Tromethamine (Tarasyn, Korea) for three days to control postoperative pain.

Histological analysis

Rabbits were sacrificed at four, eight, and 12 weeks after surgery, and calvaria were taken for macroscopic and microscopic studies. Samples were fixed in $10 \%$ neutral buffered formalin followed by decalcification via immersing in 10\% v/v nitric acid for 14 days (being refreshed every $48 \mathrm{~h}$ ). Then, dehydration was performed in a graded series of ethanol (80-100\%). Each sample was embedded in paraffin and sectioned (5 microns) by microtome (Leica Microsystems SP 1600, Nussloch, Germany) for Alizarin Red, Hematoxylin \& Eosin (H\&E) and Masson's Trichrome staining evaluations. At least, three histological sections were selected and investigated using a light microscope (Leica Microsystems AG, Wetzlar, Germany). All histomorphometric data were obtained from Image J software ( $\mathrm{NIH}$, Maryland, USA) to evaluate the new bone, residual graft, and connective tissue ( $\mathrm{n}=6$ sections/ each group).

In vitro antibacterial activity assay

Scaffolds were powdered, sterilized with Ultra Violet (UV) and added to $2 \mathrm{ml}$ Nutrient Broth (NB) bacterial culture medium at a final concentration of $175 \mathrm{mg} / \mathrm{ml}$. Each sample suspension was incubated at $37^{\circ} \mathrm{C}$ 
for 48 hours under aerobic conditions, after which the samples were centrifuged (for $5 \mathrm{~min}$ at $15000 \mathrm{rpm}$ ). One $\mathrm{ml}$ from the supernatant was transferred to sterilized tubes, and separate tubes were inoculated with each of the test microorganisms (Staphylococcus aureus ATCC28923 and Escherichia coli ATCC28922) at a final concentration of $10^{6} \mathrm{CFU}$. Free NB served as negative control, and bioglass free NB culture of $S$. aureus and E. coli were used as positive controls. The inoculated samples were incubated at $37 \mathrm{C}$ under aerobic conditions for an overnight after which samples were serially diluted and an aliquot of $100 \mu \mathrm{l}$ was cultured on Mueller-Hinton agar (MH) at $37^{\circ} \mathrm{C}$ for $24 \mathrm{~h}$. The total viable count was performed and the Colony Forming Unit (CFU) was determined on $\mathrm{MH}$ plates. The inhibition of growth was calculated as the logarithmic reduction in colony counts on $\mathrm{MH}$ plates.

\section{Statistical analysis}

All data were evaluated using the ANOVA test of SPSS (version 12.0.1, Chicago, USA). Differences were considered significant at $P$ values $\leq 0.05$.

\section{Results}

Macroscopic and Histologic Evaluation

The osteogenic capability of the prepared scaffolds was investigated by implanting Gel-BG/Sr and GelBG disks into $8 \mathrm{~mm}$ full-thickness calvarial defects after four, eight and 12 weeks. To do this experiment, rabbits were sacrificed with an overdose of anesthetics and the cranial was carefully removed. Macroscopically, the entire defect area was covered with the fibrous scar and no inflammation was observed in all experimental groups. Defects treated with scaffolds appeared thinner than the surrounding bone 12 weeks after implantation. However, those that had Gel-BG/Sr were contiguous, being hardly detectable from the nearby bone tissue with no movement (Fig. 1). For H\&E staining investigation, the samples were studied with a light microscope at magnifications of $\times 40$ and $\times 100$. Defects filled with the Gel-BG/Sr scaffolds presented immature bone development for four weeks. New bone creation was noted to begin bridging the defects at eight weeks, with noticeable cell migration. The border of the defects was hardly distinguishable from the nearby bone tissue. Although no noticeable cell permeation or new bone formation was observed in Gel-BG treated defects at four weeks, cell impregnation, new bone formation, and bridging in this group was started at eight weeks, which then were increased after twelve weeks. The unfilled defects (control group) did not heal completely, and the new bone was only noted at the defect margin with the fibrous tissue covered the rest of the defect (Fig. 2). Additionally, Masson's trichrome staining was used to confirm new bone formation (Fig. 3). For Gel-BG/Sr scaffolds, the displacement of the mature collagen at the new bone formation zone was detected. Immature bone formation was observed at four weeks, followed by the newly bone creation after eight and 12 weeks. Unlikely, in defects contained Gel-BG scaffolds as well as the control group, immature bone formation was not detected at four weeks. In defects treated with scaffolds, the newly produced bone was observed on each lateral of the margin defects as well as between the implanted scaffolds. Although in all experimental groups, the Masson's trichrome images showed the development of new collagen fibers 
(blue color), in the defects filled with Gel/BG-Sr, the number of synthesized collagen fibers was higher compared to other groups following 12 weeks post-implantation.

Mineralization stages of the formed tissue were observed by Alizarin Red staining at specific time points. As can be seen in Fig. 4, immature bone development was detected in Gel-BG/Sr at four weeks, followed by considerable new bone formation and bridging at eight and 12 weeks. For the Gel-BG scaffold, newly bone development was little, and most of the defects were clear. The new bone was only noted at the margin with fibrous tissue was observed in the rest of the defect for the unfilled defect. Our findings showed that implantation of Gel-BG/Sr could upgrade bone regeneration more successfully than Gel-BG in this model.

Histomorphometric analysis

The results of the histomorphometric analysis is presented in Table 1 during four, eight, and 12 weeks. As can be seen, the percentage of new bone was $49.10 \pm 1.03 \%, 33.88 \pm 0.35 \%$, and $17.32 \pm 0.36 \%$ for Gel$\mathrm{BG} / \mathrm{Sr}$, Gel-BG, and control groups at 12 weeks, respectively (Table 1). Bone healing was significantly increased in Gel-BG/Sr compared with other groups at 12 weeks ( ${ }^{*} \leq 05$ ). The residual graft was, also, gradually decreased in Gel-BG/Sr and Gel-BG groups reaching $41.50 \pm 0.20 \%$ and $51.34 \pm 0.06 \%$, respectively. Also, the amount of connective tissue of composite scaffolds (Gel-BG/Sr and Gel-BG) was gradually decreased during 12 weeks, while the corresponding data changes were not apparent for the unfilled groups. 
Table 1

Histomorphometric analysis of new bone, connective tissue and residual graft during 4, 8 and 12 weeks. NB: The percentage of New Bone; RG: The percentage of

Residual Graft; CT: The percentage of Connective Tissue.

\begin{tabular}{|c|c|c|c|c|}
\hline & & Gel-BG/Sr & Gel-BG & Control \\
\hline \multirow[t]{3}{*}{ NB (\%) } & 4 weeks & $11.71 \pm 0.84^{a}$ & $7.1 \pm 0.7^{b}$ & $4.2 \pm 0.42^{c}$ \\
\hline & 8 weeks & $30.2 \pm 3.3^{a}$ & $21.02 \pm 0.88^{b}$ & $14.15 \pm 0.27^{c}$ \\
\hline & 12 weeks & $49.1 \pm 3.73^{a}$ & $33.88 \pm 0.35^{b}$ & $17.32 \pm 0.36^{c}$ \\
\hline \multirow[t]{3}{*}{ RG (\%) } & 4 weeks & $60.68 \pm 1.62^{a}$ & $69.8 \pm 0.54^{b}$ & $0.00^{\mathrm{c}}$ \\
\hline & 8 weeks & $53.56 \pm 1.64^{a}$ & $58.43 \pm 1.41^{b}$ & $0.00^{\mathrm{C}}$ \\
\hline & 12 weeks & $41.50 \pm 1.94^{a}$ & $51.34 \pm 0.98^{b}$ & $0.00^{c}$ \\
\hline \multirow[t]{3}{*}{ CT (\%) } & 4 weeks & $28.15 \pm 0.73^{a}$ & $25.10 \pm 0.3^{b}$ & $95.08 \pm 0.21^{c}$ \\
\hline & 8 weeks & $16.23 \pm 0.41^{a}$ & $20.55 \pm 0.68^{b}$ & $85.85 \pm 0.56^{c}$ \\
\hline & 12 weeks & $9.4 \pm 0.18^{a}$ & $14.78 \pm 0.09^{b}$ & $82.68 \pm 0.38^{c}$ \\
\hline
\end{tabular}

Antibacterial investigations

The growth of E.coli was completely inhibited by each of the bioglass containing powder with no bacterial growth on $\mathrm{MH}$ plates. S.aureus was partially inhibited by Gel-BG/Sr, which resulted in 1000 colony-forming units (CFU)/mL on $\mathrm{MH}$ plates, equivalent to 3 log reductions in growth index. However, Gel-BG revealed no antibacterial effect on S.aureus as detected by more than $10^{6}$ (CFU)/mL growth on $\mathrm{MH}$ agar. Considering the more rigid structure of $\mathrm{S}$. aureus cell was (as a representative of Gram-positive bacteria), makes Gel-BG/Sr an achievement toward development of structures with wide-range antimicrobial efficacy.

\section{Discussion}

Aiming to enhance bone formation with less adverse systemic side effects, Sr has been recently included in many bone substitutes[14]. This ion is a trace element which induces bone development and hinders bone resorption, simultaneously[22-24]. The safety and efficacy of Sr-doped biomaterials for inducing bone formation and remodeling have significantly been reviewed[14]. In our earlier study, we also proved hat $\mathrm{Sr}$ substitution for $\mathrm{Ca}$ in Gel/BG scaffolds improves the mechanical, biological, and angiogenic properties of the scaffold[17]. In this experiment, we additionally investigated the osteogenic potential of 
Gel-BG and Gel-BG/Sr scaffolds in critically sized rabbit calvarial defects. Histological assessments were performed on the harvested scaffolds four, eight, and 12 weeks' post-surgery. No chronic inflammation was observed following implantation of the scaffolds, which confirms tissue compatibility of BG containing scaffolds[25,26]. Analysis of decalcified samples with Alizarin red staining presented much more new bone in defects filled with Gel/BG-Sr compared to those contained Gel/BG and the control groups. This indicates that $\mathrm{Sr}$ substitution in BG could remarkably increase bone regeneration capacity.

One reason that Sr promotes bone formation and remodeling could be its impact on the expression of genes, including cytokine IL-6. This cytokine, a pro-inflammatory stimulator that recruits osteoclast and induces bone reabsorption, is decreased by Sr effect[27]. Also, Sr induces genes and proteins involved in the bone formation, such as bone morphogenetic proteins and osteocalcin [28]. It has been shown that $\mathrm{Sr}$ ions can increase MSCs response as well as to hinder the differentiation of osteoclasts via inhibiting the expression of receptor activator of nuclear factor Kappa-B (RANK) ligand in MSCs [23, 29]. Besides, this ion can stimulate osteoprotegerin expression, which in turn stops the RANK and its ligand interaction, inhibiting osteoclast activity[30, 31]. It has been demonstrated that the acceleration of osteoprogenitor cells differentiation into osteoblasts could be due to the activation of membrane-bound calcium sensing receptor (CaSR) and the Wnt/ $\beta$-Catenin signaling pathway[32, 33]. Interestingly, Sr has emerged to induce angiogenic factors expression, including vascular endothelial growth factor[30]. Enhanced neovascularization caused by Sr substitution could also provide more nutrients for bone-forming cells in bone defects[19]. Therefore, activation of osteogenesis and angiogenesis could be considered as improving features for Sr containing scaffolds, as has been previously confirmed[19]. Apart from contributing factors mentioned above, the synergistic effect of the released bioactive $\mathrm{Sr}$ and $\mathrm{Si}$ ions from $B G$ is possibly another factor enhancing bone regeneration ability of the Gel/BG-Sr scaffold[19]. In a study, it was confirmed that $\mathrm{Sr}$ and $\mathrm{Si}$ in the structure of BG could synergistically activate the NFATC and Wnt/ $\beta$ Catenin signaling pathways, respectively, which in turn mediate osteogenesis[34]. Zhao et al. assessed the osteogenic capability of Sr-MBG fabricated by three dimensional (3D) printing method in critical-sized defects made in rat calvarial. Sr-MBG scaffolds exhibited superior osteocunductivity and more new vessel formation compared to MBG scaffolds for eight weeks[19].

The process of bone healing can be delayed due to the bacterial infection, which can subsequently lead to surgical failure by replacement or removal of the implanted biomaterials[35, 36].Therefore, biomaterials with anti-infective properties are required in line with the specific clinical application[37]. Many studies have indicated that BGs, even without ionic additions, have growth-inhibitory influence against several important pathogens[38,39]. Although the exact antibacterial mechanisms for the BGs remain unclear, one possible reason could be the fact that the glass sodium is being released, which is unfavorable for bacteria and increases the $\mathrm{pH}$ level. In fact, increased osmotic pressure caused by dissolution of ions, including silicon, calcium, sodium, and phosphate provides an undesirable environment for the bacteria growth. Besides, several activities in the bacterial cell, including glycolysis, trans membrane proton translocation and acid tolerance can be inhibited by dissolution ions such as zinc form BGs depending on the concentration[40,41]. Antibacterial studies of this experiment suggest that strontium substitution could increase the bactericidal effectiveness against $\mathrm{S}$. aureus and E. coli. The 
outcome was more pronounced against S. aureus. It has been reported that Sr-BGs antibacterial activity could be a result of the higher concentration of $\mathrm{Ca}, \mathrm{P}$ and $\mathrm{Sr}$ ions being released in the simulated body fluid (SBF) solutions and the higher pH values compared to the BG samples[42]. In a study by Liu et al., strontium-substituted BGs significantly inhibited the growth of sub-gingival bacteria depending on the ratio of strontium in the glasses[43]. Interestingly, the authors stated that even the base glass with no $\mathrm{Sr}$ displays an apparent antibacterial activity, which may be due to the increased amount of phosphate to 4 mol\% compared to the Bioglass ${ }^{\circledR} 45 \mathrm{~S} 5$ with $2.5 \mathrm{~mol} \% \mathrm{P}_{2} \mathrm{O}_{5}[43]$.Significant antimicrobial properties of strontium and silver-containing BG powder has also been formerly confirmed against S.aureus and E.coli bacteria. Previously, antibacterial test results have shown that the strontium substituted 58S BG could exhibit the antibacterial effect against methicillin-resistant staphylococcus aureus (MRSA) bacteria which are resistant to methicillin and other associated antibiotics of the penicillin class. Bacterial activities ,including growth and reproduction, cell wall synthesis, cell metabolism as well as chromosomal replication can be inhibited by the release of $\mathrm{Sr}^{2+}$ ions, as well[42, 44-46].

While Sr doped materials are safe and effective for stimulating bone formation and remodeling, this effect may be more noticeable over time under the concentration applied[14]. Possible factors that could affect the potential evaluation of results for Sr-enriched biomaterials activity are the rate in which Sr is being released, Sr content, experimental animal models, size, location, type of the defects, as well as the applied methods to evaluate the final response[14]. However, despite the good results, more information is required about the safety and effectiveness of local Sr usage [14]. Precautions still need to be considered as the safety of oral Strontium Renelate for the cardiovascular system could be a matter of concern $[47,48]$.

\section{Conclusion}

Bioactivity of biomaterial is an important factor to be considered as it can influence the scaffold effectiveness to induce bone formation[18]. Inclusion of $15 \mathrm{wt} \%$ of BG-Sr into a polymer bulk improved its osteogenic ability compared with that containing only BG. While retaining antimicrobial efficacy against E. coli, Sr incorporation effectively improved antibacterial activity against $\mathrm{S}$. aureus, as well.However, more detailed analysis is still required to elucidate the mechanisms of $\mathrm{Sr}$ dopant on improved bone healing for clinical applications.

\section{Abbreviations}

strontium :Sr; gelatin-BG :Gel-BG; gelatin-BG- strontium :Gel-BG/Sr; bioactive glasses :BGs; platelet-derived growth factor-BB (PDGF-BB); $p$ (N-Isopropylacrylamide-co-butyl Methylacrylate) :PIB; mesoporous bioactive glass containing Sr :Sr-MBG; human umbilical cord perivascular cells :HUCPVCs; Mesenchymal Stem Cells :MSCs; gelatin :Gel; Hematoxylin \& Eosin :H\&E;Staphylococcus aureus: S. aureus; Escherichia coli : E. coli ;Ultra Violet :UV;Nutrient Broth :NB;Mueller-Hinton :MH; Colony Forming Unit :CFU;receptor activator of nuclear factor Kappa-B :RANK;calcium sensing receptor :CaSR;three dimensional :3D;methicillin-resistant staphylococcus aureus :MRSA. 


\section{Declarations}

\section{Ethics approval and consent to participate}

Animal investigations were approved by the Ethics Committee of Tarbiat Modares University, Iran (IR.MODARES.REC.1398.070).

\section{Consent for publication}

This paper is approved by all authors for publication.

\section{Availability of data and materials}

The datasets used and/or analyzed during the current study are available from the corresponding author on reasonable request.

\section{Competing interests}

The authors declare that they have no competing interests.

\section{Funding}

This research was partially supported by Iran National Science Foundation (INSF) (Grant number: 96011120).

\section{Author's contributions}

Najafloo and $\mathrm{H}$. Eyni analyzed and interpreted the in vivo data. B. Bakhshi implemented the antibacterial experiment. N. Baheiraei designed the study and was a major contributor in writing and conducting the manuscript. All authors read and approved the final manuscript.

\section{Acknowledgements}

Not applicable

\section{Author details}

${ }^{1}$ Tissue Engineering and Applied Cell Sciences Division, Department of Hematology, Faculty of Medical Sciences, Tarbiat Modares University, Tehran, Iran. ${ }^{2}$ Department of Anatomical Science, Faculty of Medicine, Tarbiat Modares University, Tehran, Iran. ${ }^{3}$ Department of Bacteriology, Faculty of Medical Sciences, Tarbiat Modares University, Tehran, Iran. ${ }^{4}$ Department of bio-informatics, Faculty of Interdisciplinary Science and Technology, Tarbiat Modares University, Tehran,Iran.

\section{References}


1. Langer, R. and J.P. Vacanti, Tissue engineering. Science, 1993. 260 p. 920-926

2. Amini AR, Laurencin CT, Nukavarapu SP: Bone tissue engineering: recent advances and challenges. Critical Reviews ${ }^{\mathrm{TM}}$ in Biomedical Engineering 2012, 40(5).

3. Zhang Q, Chen X, Geng S, Wei L, Miron RJ, Zhao Y, Zhang Y: Nanogel-based scaffolds fabricated for bone regeneration with mesoporous bioactive glass and strontium: In vitro and in vivo characterization. Journal of Biomedical Materials Research Part A 2017, 105(4):1175-1183.

4. Marquis M-E, Lord E, Bergeron E, Drevelle O, Park H, Cabana F, Senta H, Faucheux N: Bone cellsbiomaterials interactions. Front Biosci 2009, 14:1023-1067.

5. Krishnan V, Lakshmi T: Bioglass: A novel biocompatible innovation. Journal of advanced pharmaceutical technology \& research 2013, 4(2):78.

6. Khan PK, Mahato A, Kundu B, Nandi SK, Mukherjee P, Datta S, Sarkar S, Mukherjee J, Nath S, Balla VK: Influence of single and binary doping of strontium and lithium on in vivo biological properties of bioactive glass scaffolds. Scientific reports 2016, 6:32964.

7. Jones JR: Review of bioactive glass: from Hench to hybrids. Acta biomaterialia 2013, 9(1):44574486.

8. Lin Y, Mauro JC, Kaur G: Bioactive Glasses for Cancer Therapy. In: Biomedical, Therapeutic and Clinical Applications of Bioactive Glasses. Elsevier; 2019: 273-312.

9. Nicholson J: The Use of Bioactive Glasses in Periodontology. In: Biomedical, Therapeutic and Clinical Applications of Bioactive Glasses. Elsevier; 2019: 251-271.

10. Baino F: How can bioactive glasses be useful in ocular surgery? Journal of Biomedical Materials Research Part A 2015, 103(3):1259-1275.

11. O'Donnell S, Cranney A, Wells GA, Adachi JD, Reginster JY: Strontium ranelate for preventing and treating postmenopausal osteoporosis. Cochrane Database of Systematic Reviews 2006(3).

12. Reginster JY, Felsenberg D, Boonen S, Diez-Perez A, Rizzoli R, Brandi ML, Spector TD, Brixen K, Goemaere S, Cormier C: Effects of long-term strontium ranelate treatment on the risk of nonvertebral and vertebral fractures in postmenopausal osteoporosis: Results of a five-year, randomized, placebocontrolled trial. Arthritis \& Rheumatism: Official Journal of the American College of Rheumatology 2008, 58(6):1687-1695.

13. Tenti S, Cheleschi S, Guidelli GM, Galeazzi M, Fioravanti A: What about strontium ranelate in osteoarthritis? Doubts and securities. Modern rheumatology 2014, 24(6):881-884.

14. Neves N, Linhares D, Costa G, Ribeiro C, Barbosa M: In vivo and clinical application of strontiumenriched biomaterials for bone regeneration: a systematic review. Bone \& joint research 2017, 6(6):366-375.

15. Pilmane M, Salma-Ancane K, Loca D, Locs J, Berzina-Cimdina L: Strontium and strontium ranelate: Historical review of some of their functions. Materials Science and Engineering: C 2017, 78:12221230. 
16. Molino G, Bari A, Baino F, Fiorilli S, Vitale-Brovarone C: Electrophoretic deposition of spray-dried Srcontaining mesoporous bioactive glass spheres on glass-ceramic scaffolds for bone tissue regeneration. Journal of Materials Science 2017, 52(15):9103-9114.

17. Jalise SZ, Baheiraei N, Bagheri F: The effects of strontium incorporation on a novel gelatin/bioactive glass bone graft: In vitro and in vivo characterization. Ceramics International 2018, 44(12):1421714227.

18. Poh PS, Hutmacher DW, Holzapfel BM, Solanki AK, Stevens MM, Woodruff MA: In vitro and in vivo bone formation potential of surface calcium phosphate-coated polycaprolactone and polycaprolactone/bioactive glass composite scaffolds. Acta biomaterialia 2016, 30:319-333.

19. Zhao S, Zhang J, Zhu M, Zhang Y, Liu Z, Tao C, Zhu Y, Zhang C: Three-dimensional printed strontiumcontaining mesoporous bioactive glass scaffolds for repairing rat critical-sized calvarial defects. Acta biomaterialia 2015, 12:270-280.

20. Zhao F, Lei B, Li X, Mo Y, Wang R, Chen D, Chen X: Promoting in vivo early angiogenesis with submicrometer strontium-contained bioactive microspheres through modulating macrophage phenotypes. Biomaterials 2018, 178:36-47.

21. Kargozar S, Lotfibakhshaiesh N, Ai J, Mozafari M, Milan PB, Hamzehlou S, Barati M, Baino F, Hill RG, Joghataei MT: Strontium-and cobalt-substituted bioactive glasses seeded with human umbilical cord perivascular cells to promote bone regeneration via enhanced osteogenic and angiogenic activities. Acta biomaterialia 2017, 58:502-514.

22. Bonnelye E, Chabadel A, Saltel F, Jurdic P: Dual effect of strontium ranelate: stimulation of osteoblast differentiation and inhibition of osteoclast formation and resorption in vitro. Bone 2008, 42(1):129-138.

23. Marie P, Felsenberg D, Brandi M: How strontium ranelate, via opposite effects on bone resorption and formation, prevents osteoporosis. Osteoporosis International 2011, 22(6):1659-1667.

24. Baron R, Tsouderos Y: In vitro effects of S12911-2 on osteoclast function and bone marrow macrophage differentiation. European journal of pharmacology 2002, 450(1):11-17.

25. Fialkov JA, Holy CE, Shoichet MS, Davies JE: In vivo bone engineering in a rabbit femur. Journal of Craniofacial Surgery 2003, 14(3):324-332.

26. Moimas L, Biasotto M, Di Lenarda R, Olivo A, Schmid C: Rabbit pilot study on the resorbability of three-dimensional bioactive glass fibre scaffolds. Acta biomaterialia 2006, 2(2):191-199.

27. Christoffersen J, Christoffersen MR, Kolthoff N, Bärenholdt O: Effects of strontium ions on growth and dissolution of hydroxyapatite and on bone mineral detection. Bone 1997, 20(1):47-54.

28. Rahman MS, Akhtar N, Jamil HM, Banik RS, Asaduzzaman SM: TGF- $\beta$ /BMP signaling and other molecular events: regulation of osteoblastogenesis and bone formation. Bone research 2015, 3:15005.

29. Peng S, Zhou G, Luk KD, Cheung KM, Li Z, Lam WM, Zhou Z, Lu WW: Strontium promotes osteogenic differentiation of mesenchymal stem cells through the Ras/MAPK signaling pathway. Cellular 
Physiology and Biochemistry 2009, 23(1-3):165-174.

30. Li Y, Li Q, Zhu S, Luo E, Li J, Feng G, Liao Y, Hu J: The effect of strontium-substituted hydroxyapatite coating on implant fixation in ovariectomized rats. Biomaterials 2010, 31(34):9006-9014.

31. Saidak Z, Marie PJ: Strontium signaling: molecular mechanisms and therapeutic implications in osteoporosis. Pharmacology \& therapeutics 2012, 136(2):216-226.

32. Fromigué O, Haÿ E, Barbara A, Petrel C, Traiffort E, Ruat M, Marie PJ: Calcium sensing receptordependent and receptor-independent activation of osteoblast replication and survival by strontium ranelate. Journal of cellular and molecular medicine 2009, 13(8b):2189-2199.

33. Yang F, Yang D, Tu J, Zheng Q, Cai L, Wang L: Strontium enhances osteogenic differentiation of mesenchymal stem cells and in vivo bone formation by activating Wnt/catenin signaling. Stem cells 2011, 29(6):981-991.

34. Zhang W, Huang D, Zhao F, Gao W, Sun L, Li X, Chen X: Synergistic effect of strontium and silicon in strontium-substituted sub-micron bioactive glass for enhanced osteogenesis. Materials Science and Engineering: C 2018, 89:245-255.

35. Yuan K, Chan Y-J, Kung K-C, Lee T-M: Comparison of osseointegration on various implant surfaces after bacterial contamination and cleaning: a rabbit study. International Journal of Oral \& Maxillofacial Implants 2014, 29(1).

36. Campoccia D, Montanaro L, Arciola CR: The significance of infection related to orthopedic devices and issues of antibiotic resistance. Biomaterials 2006, 27(11):2331-2339.

37. Campoccia D, Montanaro L, Arciola CR: A review of the biomaterials technologies for infectionresistant surfaces. Biomaterials 2013, 34(34):8533-8554.

38. Coraça-Huber DC, Fille M, Hausdorfer J, Putzer D, Nogler M: Efficacy of antibacterial bioactive glass S53P4 against S. aureus biofilms grown on titanium discs in vitro. Journal of Orthopaedic Research 2014, 32(1):175-177.

39. Gergely I, Zazgyva A, Man A, Zuh S, Pop T: The in vitro antibacterial effect of S53P4 bioactive glass and gentamicin impregnated polymethylmethacrylate beads. Acta microbiologica et immunologica Hungarica 2014, 61(2):145-160.

40. Echezarreta-López M, Landin M: Using machine learning for improving knowledge on antibacterial effect of bioactive glass. International journal of pharmaceutics 2013, 453(2):641-647.

41. Phan TN, Buckner T, Sheng J, Baldeck J, Marquis R: Physiologic actions of zinc related to inhibition of acid and alkali production by oral streptococci in suspensions and biofilms. Oral microbiology and immunology 2004, 19(1):31-38.

42. Moghanian A, Firoozi S, Tahriri M: Characterization, in vitro bioactivity and biological studies of solgel synthesized SrO substituted 58S bioactive glass. Ceramics International 2017, 43(17):1488014890.

43. Liu J, Rawlinson SC, Hill RG, Fortune F: Strontium-substituted bioactive glasses in vitro osteogenic and antibacterial effects. Dental Materials 2016, 32(3):412-422. 
44. Ranga N, Poonia E, Jakhar S, Sharma AK, Kumar A, Devi S, Duhan S: Enhanced antimicrobial properties of bioactive glass using strontium and silver oxide nanocomposites. Journal of Asian Ceramic Societies 2019, 7(1):75-81.

45. Enright MC, Robinson DA, Randle G, Feil EJ, Grundmann H, Spratt BG: The evolutionary history of methicillin-resistant Staphylococcus aureus (MRSA). Proceedings of the National Academy of Sciences 2002, 99(11):7687-7692.

46. Brauer DS, Karpukhina N, Kedia G, Bhat A, Law RV, Radecka I, Hill RG: Bactericidal strontiumreleasing injectable bone cements based on bioactive glasses. Journal of The Royal Society Interface 2013, 10(78):20120647.

47. Samadikuchaksaraei A, Gholipourmalekabadi M, Erfani Ezadyar E, Azami M, Mozafari M, Johari B, Kargozar S, Jameie SB, Korourian A, Seifalian AM: Fabrication and in vivo evaluation of an osteoblast-conditioned nano-hydroxyapatite/gelatin composite scaffold for bone tissue regeneration. Journal of Biomedical Materials Research Part A 2016, 104(8):2001-2010.

48. Johari B, Ahmadzadehzarajabad M, Azami M, Kazemi M, Soleimani M, Kargozar S, Hajighasemlou S, Farajollahi MM, Samadikuchaksaraei A: Repair of rat critical size calvarial defect using osteoblastlike and umbilical vein endothelial cells seeded in gelatin/hydroxyapatite scaffolds. Journal of Biomedical Materials Research Part A 2016, 104(7):1770-1778.

\section{Figures}
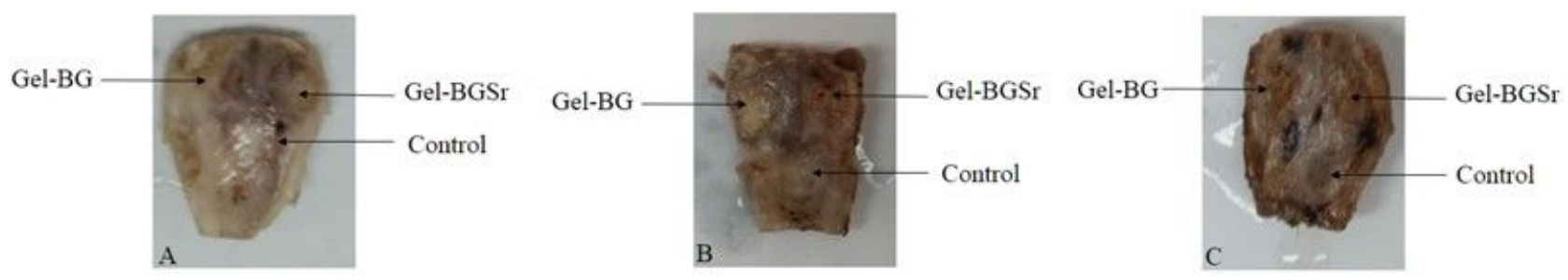

Figure 1 Macroscopic vault appearance of the defects, showing Gel-BG/Sr and Gel-BG Scaffolds and Control (unfilled) after A) 4; B) 8 and C) 12 weeks.

\section{Figure 1}

Macroscopic vault appearance of the defects, showing Gel-BG/Sr and Gel-BG Scaffolds and Control (unfilled) after A) 4; B) 8 and C) 12 weeks. 


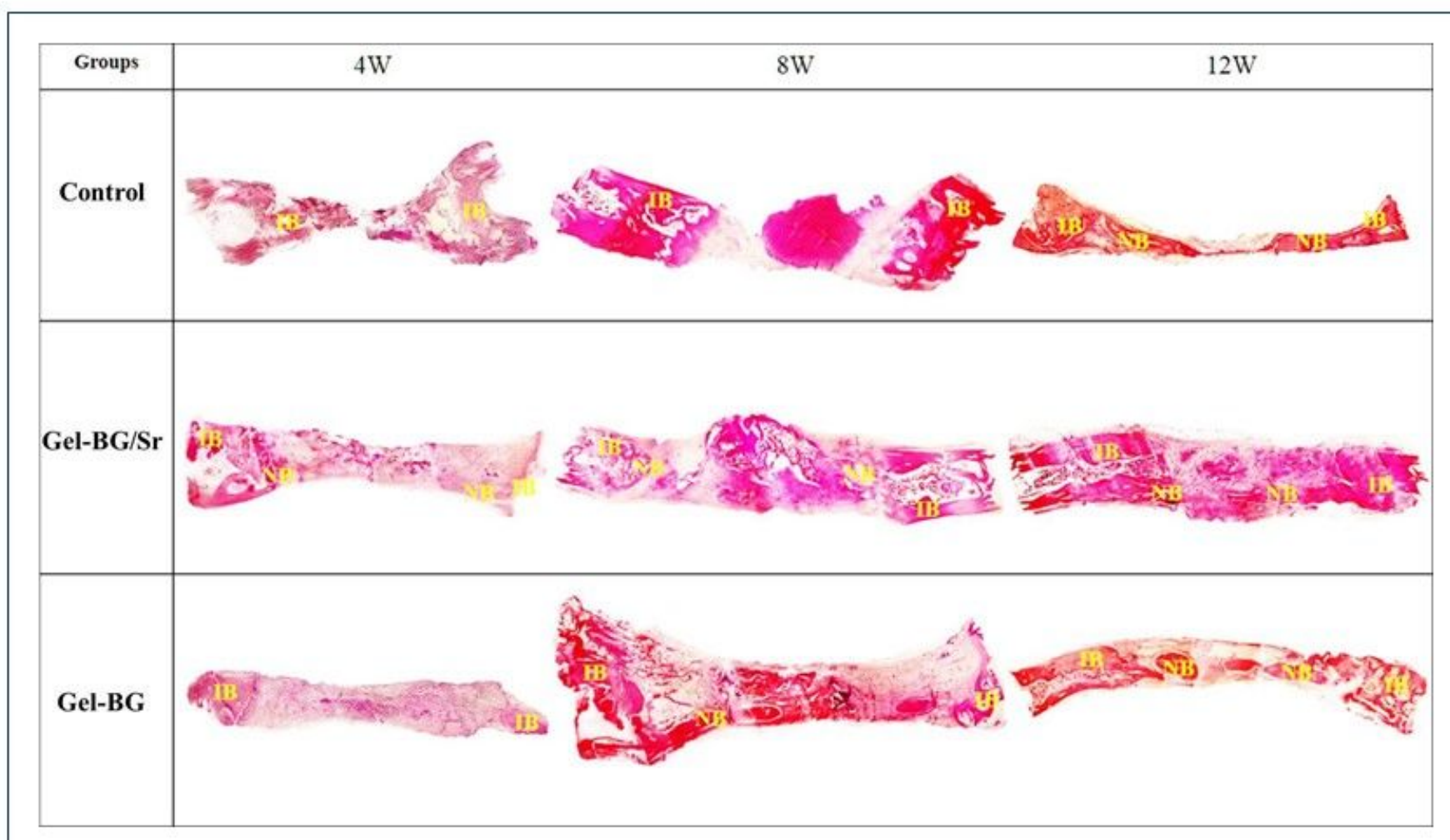

Figure 2 Histological examination (H\&amp;E staining) at 4, 8 and 12 weeks. IB( Intact Bone), NB(New Bone) (magnification $\times 40$ ).

Figure 2

Histological examination (H\&amp;E staining) at 4, 8 and 12 weeks. IB(Intact Bone) , NB(New Bone) (magnification $\times 40$ ). 


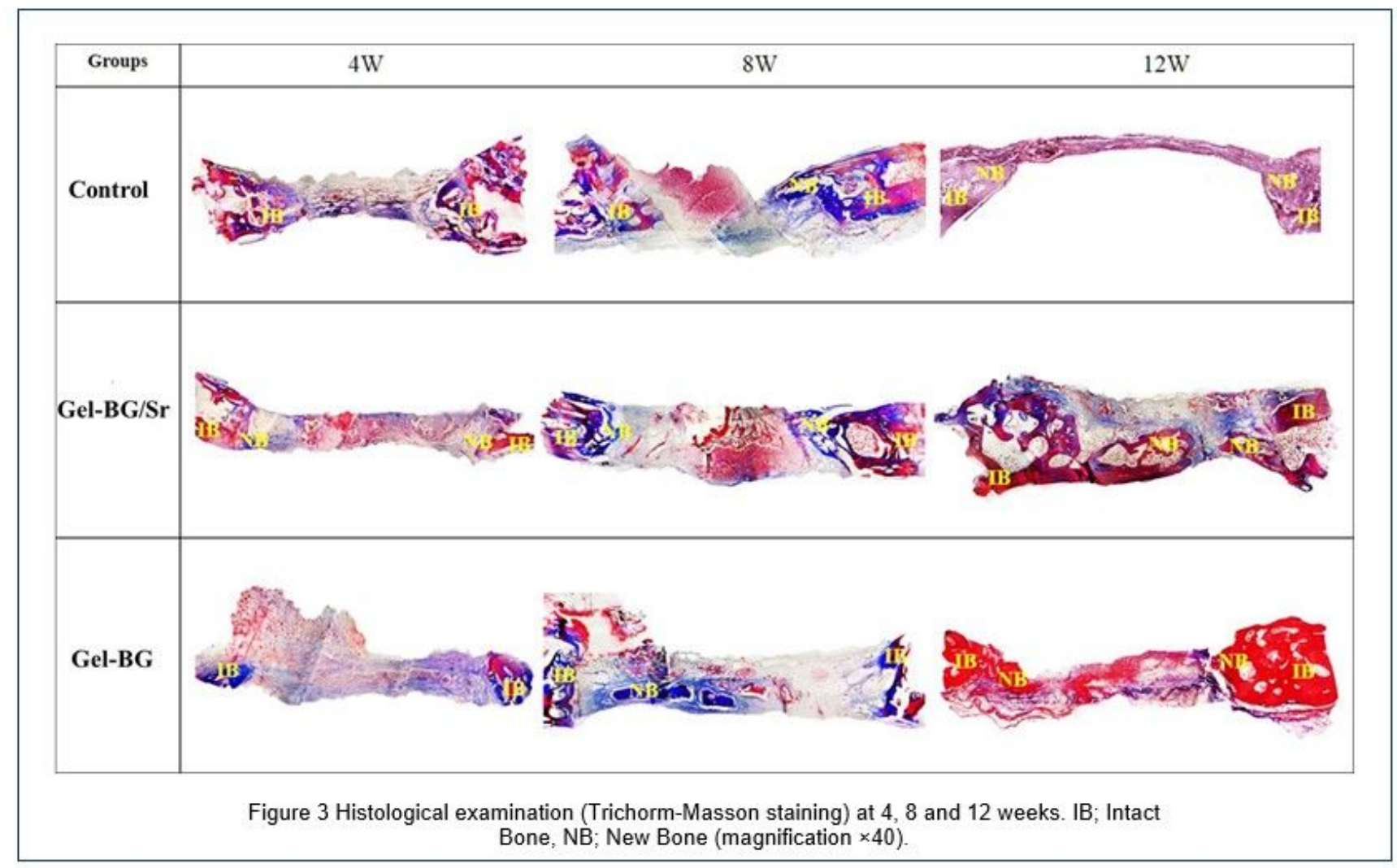

\section{Figure 3}

Histological examination (Trichorm-Masson staining) at 4, 8 and 12 weeks. IB; Intact Bone, NB; New Bone (magnification $\times 40$ ).

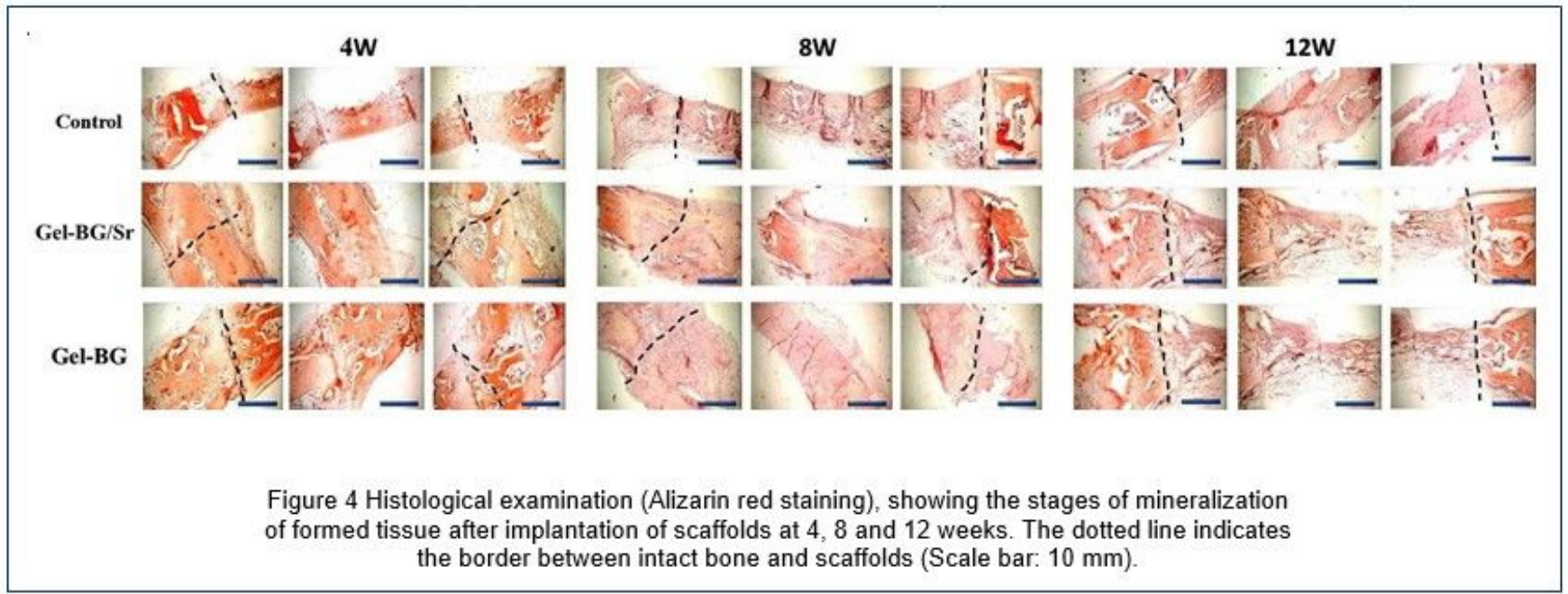

\section{Figure 4}

Histological examination (Alizarin red staining), showing the stages of mineralization of formed tissue after implantation of scaffolds at 4, 8 and 12 weeks. The dotted line indicates the border between intact 
bone and scaffolds (Scale bar: $10 \mathrm{~mm}$ ). 